\title{
Análisis de las prácticas de lectura y escritura en la formación del Historiador. Un estudio de caso a partir del análisis curricular y de la visión de los actores.
}

\author{
Daniel Eudave-Muñoz¹, Ana Cecilia Macías-Esparza², Margarita \\ Carvajal-Ciprés ${ }^{3}$
}

\author{
${ }^{1}$ Departamento de Educación, Universidad Autónoma de Aguascalientes, México | \\ deudave@correo.uaa.mx | http://orcid.org/0000-0003-4070-3109 \\ 2 Departamento de Educación Universidad Autónoma de Aguascalientes, \\ México | acmacias@hotmail.com | https://orcid.org/0000-0002-0978-752X \\ ${ }^{3}$ Departamento de Educación Universidad Autónoma de Aguascalientes, \\ México | mcarvaja@correo.uaa.mx | http://orcid.org/0000-0002-3690-7419
}

\begin{abstract}
Resumen: En los estudios de nivel superior, la lectura y la escritura son habilidades requeridas para asimilar la cultura discursiva de cada profesión. En la perspectiva de los enfoques teóricos de la Alfabetización Académica y Alfabetización Disciplinar, la formación de habilidades lectoras y de escritura debe ofrecerse a través del currículum, lo que implica partir del contexto y las situaciones formativas propias de cada carrera. En esta investigación se describe el papel que juegan las tareas de lectura y escritura en la formación del Licenciado en Historia, en una universidad pública mexicana. Se realizó un estudio cualitativo conformado por tres estrategias complementarias: análisis documental del plan de estudio de la licenciatura seleccionada, entrevistas semiestructuradas a profesores y un grupo de enfoque con estudiantes. La información aportada por profesores se complementa y contrasta con la visión de los alumnos, y estas a su vez, permiten identificar la comprensión que ambos tipos de actores tienen del currículum. Los resultados nos permiten identificar los elementos curriculares y prácticas docentes que configuran la cultura académica en este programa académico. Predominan dos tipos de tareas: a) las que están enfocadas al cumplimiento de requisitos escolares de apoyo a la docencia y la evaluación; b) las que pretenden reproducir las prácticas profesionales que los egresados habrán de enfrentar en el mundo laboral. Ambos tipos de tareas tienen como referente la meta de formar un historiador orientado a la investigación.
\end{abstract}

Palabras clave: Lectura y escritura; Alfabetización Académica; Alfabetización Disciplinar: Escritura a través del Currículum; Educación Superior.

\section{Analysis of Reading and Writing Practices in Historian Training. A Case Study Based on Curricular Analysis and Actor's Vision.}

Abstract: In higher education, reading and writing are required skills to learn the discursive culture of each profession. In theoretical views perspective of the Academic Literacy and Disciplinary Literacy, reading and reading skills development most be offered through the curriculum, which means starting from specific context and formative situations of each degree. In this research is described the role of reading and writing task in Historian degree training, in a Mexican public university. A qualitative study was made consisting of three complementary strategies: documentary analysis of the curriculum, semi-structured interviews with teachers and a focus group with students. The information provide by teachers is complemented and contrasted with student's perspective, which in turn, let identify the comprehension that both actors have of the curriculum. Results let us identify curricular elements and teaching practice that shape the academic culture in this academic program. Two types of task are dominant: a) the ones that are focus on compliment of escolar requirements of teaching and evaluation support; $b$ ) the ones that pretend replay professional practices that alumni most deal in professional world. Both areas have as referent the objective of train Historian focus on research.

Keywords: Reading and writing; Academic Literac; Disciplinary Literac; Writing across the curriculum; Higher education. 


\section{Introducción}

Las habilidades de lectura y escritura en los estudios superiores exigen más que una alfabetización básica, implican la apropiación de una cultura académica, que permita el reconocimiento de géneros y exigencias particulares de cada campo disciplinar. El desarrollo de estas habilidades especializadas puede explicarse como una alfabetización académica o como la inmersión en prácticas de literacidad. El término de alfabetización académica se refiere al conjunto de nociones y estrategias necesarias para participar en la cultura discursiva de las disciplinas, así como en las actividades de producción y análisis de textos requeridos para aprender en la universidad (Carlino 2013). Desde otros planteamientos teóricos, tenemos el enfoque de la literacidad disciplinar, el cual, "es un tipo de práctica de literacidad que se da en el marco de un dominio específico y restringido; una disciplina donde la lectura y la escritura se ven como prácticas inherentes y esenciales, las cuales pueden ser reproducidas, aprendidas y enseñadas" (Montes Silva y López Bonilla, 2017, p. 165).

Los estudios sobre literacidad disciplinar tienen un interés especial en la forma como se construyen los conocimientos al interior de cada disciplina, considerando, por lo tanto, que los procesos formativos deben reproducir, hasta donde sea posible, estas concepciones y prácticas profesionales. La instrucción formal en torno a la literacidad disciplinar, es conocida como alfabetización disciplinar: "Esta noción puede ser definida como la responsabilidad de los maestros expertos en una disciplina de compartir con sus estudiantes las formas de leer, escribir, hablar, escuchar, investigar y pensar de los miembros de esa comunidad disciplinar" (Montes Silva y López Bonilla, 2017, p. 165).

Cada programa educativo de pregrado conforma una cultura discursiva configurada a partir de diferentes tradiciones teóricas y metodológicos, el uso de géneros literarios particulares, que conllevan determinados tipos de tareas de lectura y escritura (y sus respectivas demandas cognitivas subyacentes), así como distintas maneras en que los profesores y alumnos enfrentan y resuelven las tareas de leer para escribir. Como parte de estas culturas, están también otros elementos del contexto, como son: el currículo (en sentido amplio), los apoyos institucionales, las prácticas didácticas, las tareas diseñadas explícitamente para favorecer estos aprendizajes por los distintos profesores a lo largo de una licenciatura (sin limitarse a asignaturas tales como redacción o comunicación escrita) (García Vera, 2011).

Ya sea que se aborde esta problemática desde el enfoque de la alfabetización académica, la alfabetización disciplinar o la literacidad disciplinar, los actores principales de este proceso son los estudiantes, los profesores y el contexto que construyen para lograr el desarrollo de estas habilidades. El que los alumnos reconozcan y valoren sus percepciones ayuda a la toma de consciencia sobre la importancia y el impacto presente y futuro de estas actividades en el desarrollo académico (Montijano Cabrera y Barrios Espinosa, 2016). Pero, los alumnos difícilmente podrán realizar solos un trabajo tan amplio y especializado, por lo que es indispensable el acompañamiento y la retroalimentación de parte de los profesores (Flores Aguilar, 2018).

A los docentes corresponde la definición de tareas de escritura y lectura, pero en especial, de la retroalimentación a los alumnos y su evaluación, sin limitarse a la evaluación final o calificación (Flores Aguilar, 2018). No hay que perder de vista que, como señalan Rayas Rojas y Méndez Puga (2017), más que una decisión didáctica de un docente, lo que puede dar una mayor trascendencia es la construcción de propuestas colectivas que recuperen la perspectiva de los grupos disciplinares y profesionales de referencia. 
A partir de estos elementos, se consideran los siguientes objetivos: a) identificar los momentos y espacios que en el Plan de estudio se proponen para el fomento de la lectura y escritura, en la licenciatura en Historia; b) describir el tipo de tareas de lectura y escritura, como prácticas discursivas contextualizadas, que se solicitan en los cursos de la licenciatura en Historia; c) identificar la valoración que profesores y alumnos le atribuyen a las tareas de lectura y escritura como promotoras del aprendizaje de contenidos curriculares.

El conocimiento de las propuestas curriculares y didácticas en los programas de pregrado, para la promoción de la lectura y escrituras como tareas de inserción en el campo disciplinar de cada carrera (prácticas letradas), permiten identificar aspectos que deben optimizarse para lograr el adecuado desarrollo de estas habilidades, que lo mismo tienen un impacto a lo largo de la formación universitaria, como en su futuro desarrollo profesional y en la eventual realización de estudios de posgrado.

\section{Metodología}

Esta investigación es de carácter descriptivo y muestra el contexto curricular de las tareas de lectura y escritura en un programa de pregrado, considerando el currículum en sentido amplio:

El currículum es un proceso continuo que encierra interacciones e intervenciones de orden político, ideológico, cultural, social y educativo, en sus momentos de construcción, aplicación y práctica. De esta manera, dos dimensiones sintetizan este proceso: formal, representada por el plan curricular, y real, configurada por los efectos que su práctica (del plan) ocasiona en la realidad escolar, y que no es otra cosa más que el interjuego de interpretaciones y significados, saberes e intereses que manifiestan los actores participantes en la dinámica curricular. (Ruiz, 2009: 27).

Se consideró la Licenciatura en Historia porque su perfil profesional se caracteriza en buena parte por las habilidades lectoras y en especial por las habilidades de escritura, configuradas en contextos profesionales específicos. La investigación se realizó en tres momentos, que se describen a continuación.

\subsection{Análisis Documental}

De manera inicial se realizó un análisis documental del Plan de Estudios de la Licenciatura en Historia (UAA, 2014), considerando como metodología el análisis reticular, con el propósito de identificar la composición de asignaturas y actividades que pueden aportar al desarrollo de las habilidades lectoras y de escritura. El análisis reticular o de redes, es definido por González Corzo, Martínez Cuevas, Marín Oropeza, y Bañuelos Capuchino (2010, p. 15), como "el procedimiento sistemático que tiene por objeto la descripción de la organización de los contenidos plasmados en un currículo y el establecimiento de sus relaciones de manera gráfica". En esta investigación se hizo una adaptación de la metodología de González Corzo et al. (2010), compuesta de los siguientes pasos:

1. Delimitación del universo de análisis (en este caso, el plan de estudios).

2. Análisis del perfil de egreso e identificación de los contenidos y habilidades que de manera explícita hacen referencia a la lectura y la escritura, como asignaturas, actividades o como productos.

3. Análisis de las descripciones y objetivos de las asignaturas, para identificar cualquier referencia a la lectura y la escritura, como un propósito, medio o producto. En esta descripción, se especifica el vínculo entre asignaturas. 
4. Con los elementos anteriores, explicitar las redes curriculares identificadas para el logro de la lectura y la escritura. Se elaboró un esquema en forma de red, en donde cada asignatura corresponde a un punto o vértice, el cual se integra a la red por sus relaciones con otros puntos. Las relaciones entre puntos se representan con líneas y flechas, con las que se establecen secuencias y jerarquías. Se identifican los contenidos fuente, enlace o meta (esto se explica en el apartado de resultados). En la mayoría de los casos, sólo algunos de los contenidos de cada asignatura son los que conforman la red.

Como complemento de este análisis documental se realizó una entrevista semiestructurada al jefe del departamento académico responsable de la coordinación del programa educativo, para confirmar la relevancia y rol de la lectura y la escritura en el perfil profesional, las tareas de lectura y escritura previstas en cada una de las asignaturas identificadas como claves según el análisis reticular, así como su cantidad y naturaleza. Para esta actividad se diseñó un guía de entrevista.

\subsection{Entrevistas a Profesores}

Las entrevistas a profesores se realizaron con el apoyo de una guía de entrevista que consideraba las siguientes dimensiones: propósitos de la lectura y la escritura en la formación del historiador, tipos de lecturas, géneros de escritura, actividades educativas implementadas, tipos de orientaciones y retroalimentación ofrecida a los estudiantes, dificultades que identifican en sus estudiantes. Las entrevistas fueron realizadas por los investigadores participantes y grabadas y transcritas para su análisis. Las transcripciones fueron revisadas al menos por dos investigadores para garantizar su consistencia. Se entrevistaron 5 profesores (cuatro del Departamento de Historia y una del Departamento de Letras, el cual ofrece el curso introductorio de Redacción especializada). Todos los profesores entrevistados cuentan con varios años de experiencia docente en dicho programa educativo.

\subsection{Grupos de Enfoque}

Se realizó un grupo de enfoque con nueve estudiantes del último semestre (este programa tiene una duración de 9 semestres). Como señala Vela (2013), el análisis a partir de la discusión grupal y de la estimulación colectiva de recuerdos, sentimientos y actitudes, permite recuperar con mayor fluidez los puntos de vista individuales y colectivos. Este grupo se realizó con la plataforma Meet, ya que por motivos de la pandemia del COVID 19, los estudiantes no estaban asistiendo a las aulas. Los grupos de enfoque fueron conducidos por dos de los investigadores participantes y fueron grabados y transcritos para su análisis.

\subsection{Proceso de Análisis}

Para el análisis de las entrevistas y del grupo de enfoque, se realizaron matrices agrupadas conceptualmente (Miles, Huberman, \& Saldaña, 2014), en la que las filas corresponden a cada uno de los actores educativos entrevistados y las columnas a las categorías de análisis. Las categorías de análisis se obtuvieron tanto deductivamente (a partir de las categorías de la guía de entrevista) como inductivamente (como categorías emergentes a partir de lo expresado por los entrevistados). Las categorías consideradas en el análisis fueron: propósitos de la lectura y la escritura en la formación del historiador, tipos de lecturas más frecuentes, géneros de escritura en los que se ejercitan los estudiantes, orientación sobre los criterios que deben cubrir los documentos escritos, tipo y frecuencia de la retroalimentación ofrecida a los estudiantes, principales dificultades que enfrentan los estudiantes. 
Para la integración y análisis de los resultados, se recurrió a la triangulación, por ser una estrategia acorde con los objetivos del estudio. Para este estudio, se considerarán dos tipos de triangulación, la metodológica y la de datos, siguiendo la tipología de Denzin y Lincoln (2000). Con la triangulación se busca: confirmar, complementar o refutar. Los planteamientos del plan de estudios se comparan con la visión de profesores y alumnos. De igual forma, las visiones de profesores y alumnos se comparan y confrontan, ya sea para complementar o matizar las diferentes visiones sobre las prácticas de lectura y escritura.

\section{Resultados}

\subsection{El Contexto Institucional}

El programa educativo analizado es coordinado por el Departamento de Historia, el cual se conforma por una decena de profesores de tiempo completo, que alternan sus funciones docentes con las de investigación. Algunos de ellos son fundadores de la licenciatura, que inició en el año de 1988. La línea de investigación que trabajan es la historia de México, con énfasis en la historia regional. En su mayoría cuentan con una amplia producción en investigación, consistente en libros y capítulos de libros. Profesores y egresados de este programa han contribuido a la preservación y organización de los principales archivos históricos de la entidad, los que a su vez son una fuente importante de sus investigaciones. Además de colaborar con la licenciatura, los profesores también participan en los posgrados en ciencias sociales y humanidades (maestría y doctorado) de la institución, y en ocasiones imparten cursos a otras licenciaturas.

El contexto antes descrito, favorece que los estudiantes tengan un contacto directo con actividades de investigación, además del involucramiento paulatino que como se verá en el siguiente apartado, es favorecido por el mismo plan de estudios. Es común que algunos estudiantes de la licenciatura colaboren como becarios en los proyectos de investigación de sus profesores. Desde hace poco más de una década, los estudiantes coordinan una revista en donde publican sus trabajos académicos, y en donde también participan como integrantes del comité editorial, junto con algunos de sus profesores. Aunque algunos estudiantes se inclinan más por la docencia y la divulgación de la historia que por la investigación.

\subsection{Análisis del Plan de Estudios}

El Licenciado en Historia, según el plan de estudios (UAA, 2014), es un profesionista dedicado prioritariamente a la investigación histórica, y de manera complementaria a la difusión y docencia. También se contemplan habilidades para la gestión de proyectos, archivos, organismos de difusión histórica y cultural. El énfasis en la escritura está desde el primer semestre, con la materia "Redacción especializada", que ofrece algunos principios y técnicas que habrán de perfeccionarse y complementarse a lo largo de toda la carrera. De igual forma, la lectura inicia con tres materias clave, que ofrecen técnicas propias del campo profesional: "Análisis de textos históricos", "Análisis de novela histórica" y "Manejo de fuentes" (Ver Figura 1).

Las materias que implican un mayor trabajo de elaboración de productos escritos completos y complejos, son las materias integradoras: "Taller de implementación de la historia", "Taller de Integración I y II". 
Para los Talleres de Integración, se asigna a cada estudiante un profesor-tutor (la mayoría miembros del Sistema Nacional de Investigadores ${ }^{1}$ ) para que les brinde asesoría especializada y personalizada, así como de un comité de evaluación (integrado por el profesor tutor y otros dos más). En esta licenciatura también se promueve que los estudiantes elaboren ponencias para presentar en congresos nacionales de estudiantes de este nivel.

La Figura 1 muestra las asignaturas que de manera explícita contemplan tareas de lectura y escritura y sus relaciones. Con un círculo se resaltan las asignaturas que son fuente 0 meta. Consideramos una asignatura fuente aquella que sirve de base y antecedente a una serie de asignaturas, mientras que una meta es aquella en la que se espera confluyan o se integren los conocimientos y habilidades desarrollados en otras previas. Las asignaturas resaltadas con un rombo son aquellas que sirven de vínculo entre varias. Las líneas y flechas representan los vínculos entre asignaturas, que pueden darse entre materias de un mismo semestre o como una secuencia a lo largo de varios semestres. Sólo se incluyen en la figura a las asignaturas en las que se desarrollan mayormente las habilidades lectoras y de escritura (en el entendido de que en prácticamente todas las materias se tienen que leer y escribir algo, por muy elemental que sea).

Son varios los productos que tienen que elaborar los estudiantes a lo largo de sus estudios, siendo el más relevante la tesina, producto de los talleres de integración, de la cual se espera se derive un artículo o capítulo de libro, que deseablemente tiene que ser publicado. La tesina es un género característico de las carreras humanistas (Castro Azuara y Sánchez Camargo, 2015).

\subsection{El Punto de Vista de los Profesores y los Estudiantes sobre la Lectura}

Todos los profesores de la Licenciatura en Historia valoran la lectura como parte inherente del oficio del historiador y como la principal estrategia para conocer la materia prima de su profesión (archivos, informes, textos especializados, novelas históricas, etc.). Con la lectura se espera que los estudiantes adquieran conocimientos de contenidos históricos, elementos teóricos (teorías sobre la historia y de otras ciencias sociales que apoyan a la historia), elementos metodológicos. Tres de los cinco profesores entrevistados enfatizan el análisis de comprensión (ideas, conceptos, estructura, nexos con otras asignaturas). La lectura ofrece la materia prima para el análisis de los contenidos en clase. En esta licenciatura, la clase expositiva del profesor se alterna con el análisis de lecturas hechas previamente por los alumnos, y en ocasiones con una discusión grupal.

Los profesores señalan algunas dificultades de los estudiantes: falta de hábito de la lectura; renuencia a leer textos completos (en parte porque son textos extensos y en prácticamente todas las asignaturas les encargan lecturas); dificultades para comprender los textos, por carecer de estrategias de lectura y por falta de cultura general. Los profesores reconocen la importancia de las técnicas de lectura y técnicas didácticas para favorecer la comprensión, pero dan más importancia al esfuerzo y la constancia en el trabajo de lectura. La expresión de un profesor es muy elocuente: "yo si prefiero el trabajo de músculo... fortalecer ese músculo de lectura y acostumbrarse a leer estas cosas". Por lo que señalan los profesores, se entiende que los alumnos aprenden a leer textos históricos, leyéndolos.

Los alumnos coinciden con sus profesores en que las lecturas son un complemento para el conocimiento y análisis de los contenidos de las asignaturas, y enfatizan la función crítica de la lectura (análisis crítico de los textos y de los autores revisados), aunque

${ }^{1}$ El Sistema Nacional de Investigadores es un mecanismo que reconoce la labor de las personas dedicadas a producir conocimiento científico y tecnología. Este sistema se ha implementado en México desde 1984 y lo coordina el Consejo Nacional de Ciencia y Tecnología (véase https://www.conacyt.gob.mx/index.php/el-conacyt/sistema-nacional-de-investigadores). 
comentan que no todos los profesores hacen un trabajo de análisis detallado ni todos les dan retroalimentación sobre la comprensión de dichas lecturas. Los estudiantes hacen mención de variadas actividades de lectura practicadas a lo largo de diferentes asignaturas, confirmando lo que se estipula en el plan de estudos. También coinciden con lo expuesto por los profesores en cuanto a la frecuencia de esta actvidad, una estudiante lo resume así: "Es demasiado frecuente, la base de nuestra carrera son las lecturas, nuestras fuentes primarias y sin ellas no podríamos hacer lo que hacemos. A diario las hacemos". En cuanto a las dificultades con la lectura, los estudiantes mencionan que su principal obstáculo ha sido, principalmente en los primeros semestres, los tecnicismos y conceptos especializados, que les costaba trabaja comprender. Algunos profesores les brindaron diferentes apoyos para superar estas dificultades, pero otros sólo les solicitaban las evidencias de la lectura. Los estudiantes reconocen la relación existente entre lectura y escritura, un joven los menciona así: "La escritura es como el pasto propio de la lectura, una lectura puede quedar incompleta si no hay una escritura que sintetice o que genere una parte del conocimiento tanto de tu propia cosecha como de las ideas que propone cada autor, creo que es algo necesario e ideal; va de la mano con la lectura para poder plantar tus propios cuestionamientos".

\subsection{El Punto de Vista de los Profesores y los Estudiantes sobre la Escritura}

Al igual que con la lectura, en esta carrera se aprende a escribir escribiendo y a lo largo de toda la carrera y en la mayoría de las asignaturas. Un profesor lo expresa de esta manera:

"la escritura no viene de la nada, sino de un trabajo de lectura, un trabajo de reflexión y un trabajo de ejercicio constante, y sobre todo pensar la estructura misma de la escritura, que mis ideas queden claras y que tengan una argumentación suficientemente clara, una redacción clara y precisa, al menos eso pretendo, y que tenga las fuentes de modo tal que permitan justificar eso."

Tener la capacidad de escribir un artículo o un libro, señala un profesor, "es cuestión de sobrevivencia para el historiador", por tanto, se ofrecen diferentes orientaciones y apoyos a lo largo de la carrera. Al igual que las lecturas, la escritura se solicita de manera constante en las asignaturas de esta carrera, siendo los trabajos escritos el principal elemento para evaluar a los estudiantes. A los alumnos se les dan orientaciones generales de como presentar un escrito: extensión, estructura, presentar ideas principales, citar fuentes, etc. También se comentan o exponen los criterios que debe cubrir un texto histórico, ejemplificando con algún texto modelo, con énfasis en los textos de investigación, dejando en segundo plano los de divulgación y didácticos. Algunos profesores ofrecen guías puntuales de lo que esperan como trabajo escrito.

En las asignaturas que implican la elaboración de un texto extenso (ensayo, investigación), los profesores mencionan que a los alumnos se les solicitan avances a lo largo del semestre, para revisarlos y corregirlos, señalándoles los aspectos que se deben corregir (ya sea la claridad de las ideas, la congruencia entre ideas, su orden y jerarquía, ortografía, sintaxis, etc., así como elementos metodológicos, como la claridad de los objetivos, los alcances de las preguntas de investigación, su justificación, etc.). Los profesores coinciden en que la mayoría de los estudiantes presentan muchas dificultades para redactar, al inicio de la carrera no saben cómo expresar sus ideas, tienen muchas faltas de ortografía, aunque consideran que finalmente la mayoría de los estudiantes logra desarrollar las habilidades mínimas de escritura.

Los estudiantes coinciden en que la escritura en el campo de la historia es el principal medio para comprobar y compartir el conocimiento. Están de acuerdo en que, al escribir, deben pensar en dos tipos de lectores, uno especializado y el otro, un público más amplio. Una estudiante lo resume así: "Debe existir un equilibrio para que lo entienda tanto alguien académico y alguien no académico, nuestra misión es divulgar la historia de una manera comprensible que puedan entender todas las capas de la sociedad". 
Reconocen la importancia de que sus escritos estén bien fundamentados en diferentes fuentes (archivos, textos, testimonios escritos y orales, etc.), con lo que dan énfasis a la investigación histórica.

En cuanto a las orientaciones sobre lo que debería tener un trabajo escrito, comentan que no hay una manera uniforme de hacerlo, pues si bien algunos profesores dan orientaciones precisas, otros sólo hacen referencia a los aspectos de forma o a ninguna en particular. Así mismo, la retroalimentación recibida a sus escritos depende de la disposición de cada profesor (algunos sí ofrecen comentarios detallados y sobre aspectos de contenido, congruencia de ideas, formato, ortografía, etc.).

Entre las dificultades que los estudiantes mencionan que enfrentan con la escritura, está el procesar y sintetizar toda la información que obtienen en sus investigaciones, el encadenar ideas y oraciones, citar las fuentes, etc. Resulta interesante que se resaltara como una dificultad el estado de ánimo, pero tal vez esto se deba a la situación de confinamiento que enfrentaron prácticamente durante todo el año 2020 (los grupos de enfoque se realizaron en noviembre de 2020), y a la imposibilidad de trabajar en espacios más adecuados, como las bibliotecas y los archivos históricos. Ante esta contingencia, mencionan el apoyo que les han brindado algunos profesores proporcionándoles materiales adicionales.

\section{Conclusiones}

La aspiración formativa expuesta en el perfil profesional y las redes identificadas en la malla curricular del plan de estudio de la Licenciatura en Historia permite configurar los elementos de una cultura académica particular, en donde la lectura y la escritura (en especial la última), son prácticas instituidas e instituyentes de la cultura académica (García Vera, 2011). La cultura discursiva de esta carrera está configurada por los propósitos, prácticas, productos específicos, y todo un conjunto de acciones institucionales que dan apoyo y sirven de controles o elementos de supervisión.

En el programa educativo estudiado, no se fomenta la lectura y la escritura como una habilidad inicial o básica, sino como prácticas de literacidad disciplinar, esto es, atendiendo a requerimientos de un contexto profesional específico, con exigencias discursivas propias de la formación disciplinar, que pretenden aproximar a los alumnos al desempeño profesional, a las actividades investigativas y a asumir el rol de autores y evaluadores de sus propios textos y de sus compañeros (Sánchez Upegui, 2016).

Lo planteado como propósito formativo en el plan de estudios (dimensión formal del currículum) es llevado a la práctica por los profesores, si bien, con diferentes niveles de detalle y con diferentes tipos de acompañamiento a los estudiantes (dimensión real del currículum), y de esto dan cuenta las aportaciones de los profesores entrevistados y los estudiantes que participaron en el grupo de enfoque. El acercamiento metodológico utilizado permitió tener una visión amplia de la propuesta educativa analizada.

El conocimiento de las propuestas curriculares y didácticas en los programas de pregrado, para la promoción de la lectura y escrituras como tareas de inserción en el campo disciplinar de cada carrera, permite identificar aspectos que deben optimizarse para lograr el adecuado desarrollo de estas habilidades, que lo mismo tienen un impacto a lo largo de la formación universitaria, como en su futuro desarrollo profesional y en la eventual realización de estudios de posgrado. 
Vol. 7 | Investigación Cualitativa en Educación: Avances y Desafíos

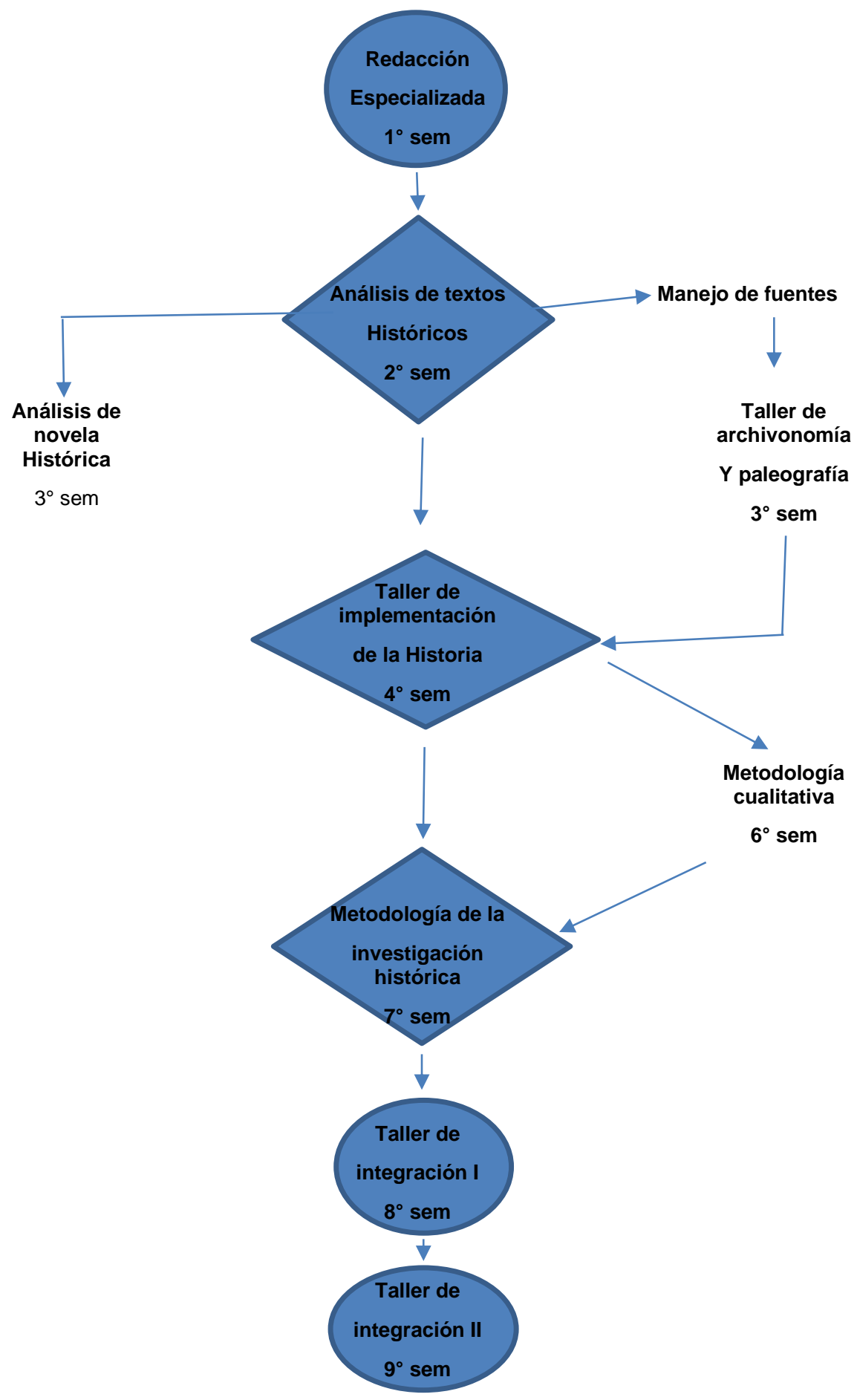

Fig. 1. Redes en el mapa curricular de la Licenciatura en Historia.

Fuente: elaboración propia. 


\section{Agradecimientos}

La investigación se realizó con recursos del Programa de Investigaciones Educativas de la Universidad Autónoma de Aguascalientes. Agradecemos a Martha Cinthia García Gaytán y Estefany Mariela Hurtado Sedano, por el apoyo brindado durante el trabajo de campo y el proceso de análisis.

\section{Referencias}

Carlino, P. (2013). Alfabetización Académica diez años después. Revista Mexicana de Investigación Educativa 2013, 18 (57) Disponible en www.redalyc.org/articulo.oa?id=14025774003

Castro Azuara, M.C. y Sánchez Camargo, M. (2015). Escribir en la universidad: la organización retórica del género tesina en el área de humanidades. Perfiles Educativos, XXXVI (148), 5067, disponible en: http:// www.iisue.unam.mx/perfiles/perfiles articulo.php?clave=2015-148-5067 (consulta: 15 de junio de 2020).

Denzin N, Lincoln Y, (Editores) (2000). Handbook of qualitative research, 2nd ed, Thousand Oaks: Sage Publications

Flores Aguilar, M. (2018). La escritura académica en estudios de ingeniería: valoraciones de estudiantes y profesores. Revista de la Educación Superior 47 (186), 23-49.

García Vera, N.O. (2011). Cultura académica y prácticas de lectura y escritura, a propósito de la formación universitaria de docentes. Pedagogía y Saberes, 35, pp. 117-139

González Corzo, E., Martínez Cuevas, P., Marín Oropeza, D.I., y Bañuelos Capuchino, J.R. (2010). Red curricular: una herramienta para el diseño de instrumentos de evaluación. Cuaderno Técnico 5. México: CENEVAL

Miles, M.B., Huberman, A.M., \& Saldaña, J. (2014). Qualitative Data Analysis. A Methods Sourcebook ( $3^{\circ}$ ed.). Thousand Oaks, CA: Sage

Montes Silva, M.E. y López Bonilla, G. (2017). Literacidad y alfabetización disciplinar: enfoques teóricos y propuestas pedagógicas. Perfiles Educativos, XXXIX (155), 162-178

Montijano Cabrera, M., y Barrios Espinosa, M. (2016). Alfabetización académica en la universidad: percepciones de estudiantes sobre una tarea escrita. Opción, 32 (8), 289-309.

Rayas Rojas, L., y Méndez Puga, A. (2017). Los estudiantes universitarios ante la escritura del ensayo académico: dificultades y posibilidades. Innovación Educativa, 17 (75), 123-147.

Ruiz, E. (2009). Propuesta de un modelo de evaluación curricular para el nivel superior. Una orientación cualitativa, México: UNAM-IISUE

Sánchez Upegui, A.A. (2016). Alfabetización académica: leer y escribir desde las disciplinas y la investigación. Revista Lasallista de Investigación. 13 (2), pp. 200-2009

UAA (2014). Plan de Estudios de la Licenciatura en Historia 2014. México: Centro de Ciencias Sociales y Humanidades, Universidad Autónoma de Aguascalientes

Vela, F. (2013). Un acto metodológico básico de la investigación social: la entrevista cualitativa, en Tarrés, M.L. (Coordinadora) Observar, Escuchar y Comprender. Sobre la tradición cualitativa en la investigación social, México: El Colegio de México-FLACSO 